\title{
A Quantitative Analysis on Network Communication Research Papers in China from 1995 to 2006
}

\author{
Chengliang Zhang \\ Ludong University \\ Yantai 264025, China
}

Tel: 86- 535-6672149 E-mail: gongge_zhang@sina.com

\begin{abstract}
In recent years, the research on network communication becomes popular gradually along with the upsurge of new media, and turns into a new focus in new communication field. By studying network communication papers from 1995 to 2006, this paper makes a relatively objective quantitative analysis of network communication's present researches and latest explorations in China.
\end{abstract}

Keywords: Internet communication research, Quantitative analysis, Research subject

Network communication is the information communication activity of human being by computer net. It takes the network communication five years to catch up with the audience scale of broadcasting and television, which have spent 38 years and 13 years respectively on reaching the same audience scale. As a brand-new modern communication method, network communication has already become an irreplaceable media. Along with the fast development of network communication, relevant research has gradually become a hot in domestic academic field.

\section{The background of research and selection of samples}

Accompanied with the upsurge of new media, researches on network communication tend to be prosperous and colorful recently. Some researchers think that: "The year 2006 is right for further development after Internet entering a new stage in China. The year 2006 is the first year of implementing the "eleventh five-year plan". China network media and network communication display an unprecedented vitality and develop very well. Meanwhile, the Internet develops forward in a rational and normative way. (Dahong Min, 2006)" Compared with the practice of network communication, present researches lag behind and most focus on the application. Corresponding summarized qualitative and quantitative researches will undoubtedly drive the development of network researches, and put forward new requirement for constructing the network communication theory system as well.

Among all knowledge carriers for research fruits of social sciences, journals are extremely important information sources. Papers in journals serve as mirrors imaging trends and development of scientific research. Therefore, studies of papers on network communication in journals can reflect the present researches on network communication. In this paper, the author targets on the authoritative retrieve journal Chinese Journal Full-text Database and collect all theses with the subject "network communication" from 1995 to 2006 by an exact retrieve. Then the author gets 3044 qualified theses. Deleting the consultant and service information, the author finally establishes 2695 sample theses.

2. Statistic of network communication research papers' time and space span and researchers and relevant analyses

\subsection{Statistic and analysis of time span}

Since Internet enters China in 1994, it has developed 13 years. Network communication research has begun from 1995 and gradually become an important part of news information communication. This paper selects the time period from 1995 to 2006 to make statistic analysis. In 12 years, there are 2695 effective research papers, annually 224.8 papers being published.

The figure 1 and table 1 shows the trends of numbers: from 1995 to 1999, researches on network communication just began. Most papers during this period are discussions and examples of institutional management. From 1999 to 2000, researches were rising sharply. In 2000, 232 papers were published, increasing 2.7 times than last year. The year 2000 became an important signal for annual network communication research. After 2002, researches slowed down and started adjustments, but began a slow rise in later three years. From 2005 to 2006, papers increased fast. In 2006, 
network communication research became a most active field in China news communication studies. In this year, 536 network communication papers were published, being 2.39 times of the annual average, which was the top during these years. Based on the time periods of researches, some scholars divide the network communication from 1996 to 2006 in China into three stages: the starting stage (1996-1999), the upsurge stage (2000-2002), and the deep development stage (2004-2005) (Li Zhao, 2006). The division of the three stages is basically in accord with the numbers of papers on network communication research.

Based on the three stages, in 2006 network communication research papers flourish again and enter an active stage after the deep development. In the research method aspect, the network communication research in 2006 is still mainly qualitative studies. And more quantitative studies appear comparing with former periods. Because of the global nature of network communication, researchers mostly adopt a "comparison" perspective to study network communication (Lidan Chen \& Yuhui Fu, 2007).

The publication trends of network communication research papers prove that the development of network, as a new thing, follows Rogers' $S$ shape innovative spreading of new thing (the new thing develops slowly at the spreading stage, then grows faster and faster, and finally enters the stable development stage), and the development law of new science from slowness to rapidness, from bottom to top, from face to depth (Xi Yao \& Mingliang Li, 2005).

The publication of China network communication research papers is in accord with the background of times. China Internet Network Information Centre (CNNIC) issued the Statistic Report for China Internet Network Development in 1997 for the first time. At that time, China Internet had 620,000 users. In late 2000, the number reached 22.5 million (Wenbo Kuang, 2001). According to the statistic data of Internet users, the rise of users directly drives the development of network communication research, which proves too much attention on practices in the research. Scholars unconsciously follow the exterior needs of Internet development. However, due to lacking of independent theoretical studies, there is a gap between practices and rationalities in network communication research. Rationality comes from long-term practices. It results from accumulation and inspiration of experiences and facts (Jianming Liu, 2006). The development speed and scale of Internet frustrate the network communication researchers. And catching-up researches can not serve as accumulations for rationality. Therefore, in a scientific sense, present network communication research is far from maturity.

\subsection{Statistic and analysis of Journals that publish relevant research parpers}

After getting necessary data resources, the author summarizes the spatial distribution of network communication research papers published in Chinese Journal Full-text Database from 1995 to 2006 according to types of journals, hoping to analyze the journal resources of network communication research, understanding the features of these journals, and identifying the statistic problems in network communication research.

Data in table 2 shows that journals of colleges and universities serve as the main stage for network communication research papers. 429 journals of colleges and universities account for $43.95 \%$ of total journals that publish network communication research papers. Although journals of colleges and universities and relevant papers distribute separately, only 1.69 papers per journal in average, the number of total papers reaches 727 , accounting for $26.98 \%$ of total relevant papers, holding a leading position. In contrast to other specialty journals, journals of colleges and universities are mostly about integrated social sciences. They offer an academic platform for different specialties, which benefits the network communication research as well. Journals of colleges and universities publish the largest number of relevant papers, which indicates that the network communication research is chiefly performed by colleges and universities. And the researchers in colleges and universities are mainly teachers and members in communication departments, libraries, and journals. The dispersion of network communication researchers is quite evident.

News communication journals are also an important stage for publishing network communication research papers. Although only 32 journals are involved, accounting for $3.28 \%$ of total relevant journals, they publish 613 relevant papers, accounting for $22.75 \%$ of total relevant papers (see figure 2 and 3). Especially, the average reaches 19.16 papers per journal.

Along with the electric development of books and archives management, journals of library, archive, and information becomes a place for publishing network communication papers. In this field, 45 journals publish 339 cross-subject papers totally. It means the network communication research has been implemented widely in different fields.

Because network communication has close connection with broadcasting, television, and publication, this kind of journals also serve as an important stage for publishing network communication papers. Relevant papers published by these journals account for $9.09 \%$ of the total relevant papers.

As network communication research develops prosperously today, many network information technology journals come into being, serving as an irreplaceable power in network communication research. Data shows that there are totally 231 network communication papers published in 68 network information technology journals. 
Because network communication is a new comprehensive frontier science, lots of research papers are separately published in other specialty journals, such as sociology, economics, politics, education, science \& technology, and culture. Science \& technology education is an important place for practice of network communication research. Journals in this field totally publish 179 relevant papers, accounting for $6.64 \%$ of the total. Sociology and economics have begun to notice the development of network communication in recent years. $2.24 \%$ of relevant papers are published in this kind of journals. Besides, 203 journals of law, medicine, and other social sciences publish 220 relevant papers, merely accounting for $8.16 \%$ of the total. And the average is only 1.08 papers per journal. It proves that there is certain dispersion for the publication of network communication research papers in journals of social sciences.

By analyzing research samples generally, the author also finds that 976 journals related with network communication research totally publish 2695 relevant papers and the average is 2.79 papers per journal. It indicates that the spatial distribution of China network communication research papers has higher dispersion in recent twelve years. That is derived from the comprehensive and frontier nature of network communication research. Data shows that among those new network communication journals, no core journal can shoulder the responsibility of supporting the academic status of network communication.

\subsection{Structure of researchers}

The structure of researchers concerns statuses and places where they work (Li Zhao, 2006). Here based on requirements of research, the author collects data of researchers' statuses. By a random selection from 2695 papers, the author takes 500 papers as samples for analyzing the structure of researchers. Data shows that authors of 409 papers are teachers, students, and researchers from colleges and universities, accounting for $81.8 \%$ of total authors of 500 samples. It indicates that network communication researchers are mostly from the institution school. 54 authors are from news media field, accounting for $20.8 \%$ of the total. Although most authors are from the institution school, it is obvious that under the background of media convergence, to probe into the convergence and interaction of media is a trend. In addition, 37 researchers are from medicine, finance, and other social agencies, accounting for $7.4 \%$ of the total. It enhances the dispersion of network communication research and means that the integrated application of network communication comes into being.

The development of any specialty connects closely with excellent experts in the field, and amounts of core authors as well. The so-called "core authors" write more papers and are more influential. Based on statistical data, the author gets the table 3, showing the situation of authors issuing network communication research papers.

According to data of network communication research papers, 2695 papers have 2174 first-authors. The average is 1.24 papers per first-author. 6 first-authors issue more than 10 papers. Dahong Min, the director of the Network and Digital Medial Lab, News and Communication Research Center, China Academic of Social Sciences, issued 21 network communication research papers in journals of news and communication in these years. His papers serve as important references for network communication research. Besides, Lidan Chen, Yu Hu, Wenbo Kuang, Ke Qin, and Xinxin Deng also issue more than 10 network communication research papers. All of them are active scholars in network communication field from colleges and universities. In a sense they are the "core authors" for network communication research.

1883 authors issue only one relevant paper, accounting for $86.61 \%$ of the total. The proportion is higher than the classical reference value $60.79 \%$ in Lotka's Law (Xi Yao \& Mingliang Li, 2005). It indicates that the network communication research has already gained wide attentions from academic field due to the popularity of network communication today.

\section{Analyze the research method and research subject}

\subsection{Statistical analysis of research method}

Network communication changes traditional mass communication mode. It brings about many new subjects for the research method of communication (Yan Chen \& Jinghong Wang, 2003). Most network communication research papers adopt the qualitative research method. The qualitative mode usually proves a theory or fact by citing classical theories, others' opinions, and history materials. In contrast, the new arisen quantitative research method is to measure the objective fact quantitatively and draw practical conclusions by mathematical induction and analysis (Chunwei Liu, 1997). The network communication emphasizes on practicality. It has mutual connections with education, science \& technology management, library and information. Comparing with other social sciences, it should focus more on quantitative researches. Although numerous papers are about network communication research, most papers are general descriptions or introductions of network communication according to experiences. In these sample papers, only 64 papers are quantitative or semi-quantitative researches based on data analysis, merely accounting for $2.37 \%$ of the total samples. It means that the research method of network communication is too simple. Researchers are not good at illustrating their points by data analyses. Surely, it is the nature of social sciences that determines the condition. But it does prove a fact that most researchers are incapable of making best use of data models due to their academic 
background and poor mathematical abilities. The research method is too abstract and lack of preciseness (Xi Yao \& Mingliang Li, 2005).

In recent years, the development of network communication research, especially the emergence of interdisciplinary researches in semiotics, information, and information management greatly enriches the scope of network communication research and also makes qualitative and semi-quantitative research possible in network communication, which significantly changes the single research method of network communication research. Since 2003, quantitative and semi-quantitative research papers rises to a great degree. From 2003 to 2006, quantitative research papers account for $42.5 \%$ of the total papers published during this period.

In 2006, the concept of Internet micro content arouses attentions of academic field. Some researchers think: any data generated by Internet users can be taken as micro content. These separate and messy data collect together and form the source of network magic power. As a tool appears (such as Google, a representative of its first-generation product), micro content steps in the stage of social development and becomes a leading actor in creating history (Guoming Yu, 2006). The micro content concept offers theoretical support for the data collection of network communication's quantitative research. In a practical aspect, data collection of network communication becomes normal gradually: CNNIC and relevant research institutions issue network data periodically; the Network Data Analysis and relevant works appear in the network informetrics research field. That offers data analysis and support for researchers' quantitative research (Tingxiao Wen, 2005).

\subsection{Statistical analysis of research subject}

To analyze the research subjects of papers, the author adopts the method used in Li Zhao's Progresses and Shortcomings of China Network Communication Studies in Ten Years (Li Zhao, 2006), classifying the research subjects into nine types: network media, network news workers, network ethics and regulations, network communication education, macro and micro effects, website construction and operation, network audience, network content analysis and others.

The table 4 displays the distribution of research subjects. Network media papers occupy the largest percentage, reaching $23.67 \%$. A specific network media subject includes not only effects of network media on mass communication but also the convergence and competition of media. In 2006, the convergence of traditional media and new media becomes a trend of researching the media development. 36 papers are about this subject. Besides, $20.82 \%$ of papers concern network ethics and regulations, which connect closely with some network cases appeared recently. Papers of network news workers, network construction and operation also occupy certain percentages. The occupation ratios exceed 10\%.

\section{Conclusion}

After more than ten years' development, more and more network communication research papers have been published. Lots of scholars with different scientific background and in different industries devote themselves to the research and achieve noticeable progresses. However, many problems appear at the same time. This paper tends to put forward these problems by quantitative research.

(1) The distribution of papers shows a high dispersion and there is not a core orderly academic stage for network communication research. Although lots of authors focus on this field, the widely-accepted "core authors" are not popular yet. And most research papers are based on experiences. Similar to the theoretical research of journalism, few researches are about fundamental theories of network communication. A basic research construction from perspectives of psychology and sociology does not come into being. If take references from the interdisciplinary research fruits and methods of information science, semiotics, hermeneutics, ecology, psychology, and communication, the network communication research will realize an more effective development.

(2) Researchers are short of academic freedom. They emphasize on qualitative research instead of quantitative one. In contrast to the fast development of network communication, researchers show an extremely passiveness. They just explain the network communication, as a new thing, and relevant concepts mechanically. Purposeful researches are very few. Present researches are chiefly about application, neglecting the construction of basic theories. Especially in recent two years, along with the development of case studies, some researchers rush into this field. It is a kind of passive response to news and cases. The research method tends to be simple and micro.

(3) Team research and specialty operation wait for further improvement. Among those network communication research papers, seldom are completed by project teams. Surely, it is caused by the contradiction between network development speed and project application procedures in a sense. Besides, interdisciplinary cooperative researches are few. Although there is interdisciplinary research, it limits to one discipline. Interdisciplinary borders are still there. Independent researches can not form an integrated power in a short period, which affects the quantitative research either.

(4) Researchers think a lot of timeliness but neglect data and accumulation of practical researches. According to the distribution of research subjects, amounts of research papers have been published during the first year when network new thing appears. In 2006, researchers pay attentions to "blogs", "exchangers", and "sharers". Once a hot subject is not 
popular any longer, relevant research papers decrease sharply. Researchers turn into next interesting research subject immediately. That is caused by not only subjects of journals but also the irrational academic atmosphere. Driven by the network development, researchers just rush into the new subject but no time to research one specific subject deeply. As a result, network communication research tends to be separate and incoherent.

\section{References}

Chen, Lidan \& Fu, Yuhui. (2007). Prosperous and active network communication research ------ a summarization of 2006 China network communication research. Contemporary Communications, No.1.

Chen, Yan \& Wang, Jianghong. (2003). Network communication: puzzles and thoughts on research methods. Contemporary Communications, No.1.

Kuang, Wenbo. (2001). A quantitative research on network audience. Journal of International Communication, No.6.

Liu, Chunwei. (1997). Implementation of quantitative analysis in news. China Radio \& Academic Journal, No.1.

Liu, Jianming. (2006). The scientific crisis of news theory. Journal of International Communication, No.9.

Min, Dahong. (2006). 2006 China Internet develops forward in a rational and order way. Media, No.12.

Wen, Tingxiao. (2005). The news breakthrough of network communication and network informetrics ------ a comment on Professor Junping Qiu's Network Data Analysis. Library and Information Service, No.6.

Yao, Xi \& Li, Mingliang. (2005). A quantitative analysis of advertising studies in China mainland. [Online] Available: http://journal.whu.edu.cn/research/read_research.php?id=295.

Yu, Guoming. (2006). Convergence and exploration of micro contents: technological keys for contents production of network media. Network Communication, No.1.

Zhao, Li. (2006). Progresses and shortcomings of China network communication studies in ten years. Journal of International Communication, No.11.

Table 1. Network communication research papers published from 1995 to 2006.

\begin{tabular}{|l|l|l|l|l|l|l|l|l|l|l|l|l|l|}
\hline Year & 1995 & 1996 & 1997 & 1998 & 1999 & 2000 & 2001 & 2002 & 2003 & 2004 & 2005 & 2006 & Total \\
\hline Number & 4 & 6 & 6 & 24 & 86 & 232 & 323 & 393 & 329 & 367 & 389 & 536 & 2695 \\
\hline Percentage (\%) & 0.15 & 0.22 & 0.22 & 0.89 & 3.19 & 8.61 & 11.99 & 14.58 & 12.21 & 13.62 & 14.43 & 19.89 & 100 \\
\hline
\end{tabular}

Table 2. The distribution of network communication research papers in different kinds of journals.

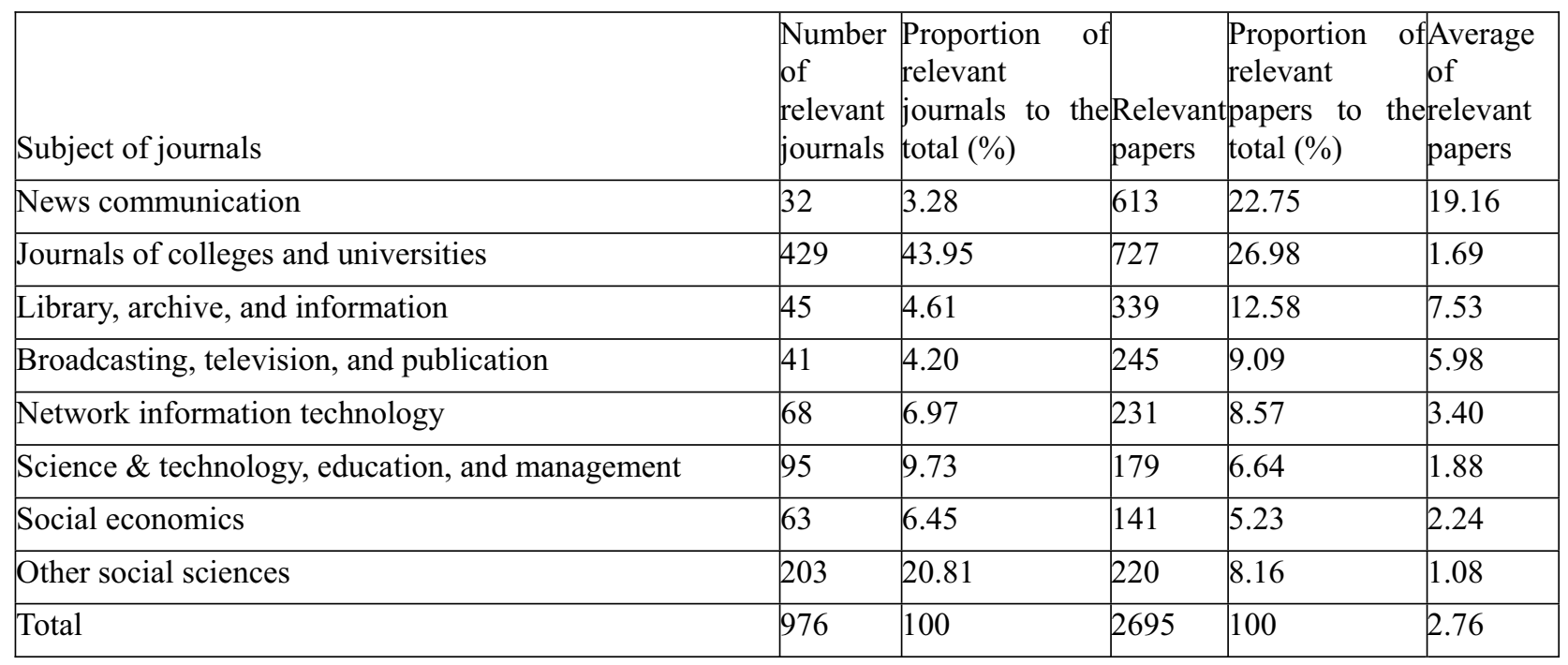

Table 3. The situation of authors issuing network communication research papers.

\begin{tabular}{|l|l|l|l|l|l|l|l|l|l|l|}
\hline Number of papers & 1 & 2 & 3 & 4 & 5 & 6 & 7 & 9 & 10 above & Total \\
\hline Number of authors & 1883 & 198 & 52 & 17 & 7 & 8 & 2 & 1 & 6 & 2174 \\
\hline $\begin{array}{l}\text { Proportion of certain kind of } \\
\text { authors to the total (\%) }\end{array}$ & 86.61 & 9.11 & 2.39 & 0.78 & 0.32 & 0.37 & 0.09 & 0.05 & 0.28 & 100 \\
\hline
\end{tabular}


Table 4. Subjects of network communication research papers.

\begin{tabular}{|l|l|l|}
\hline Subject & $\begin{array}{l}\text { Number } \\
\text { papers }\end{array}$ & of \\
\hline Network media & 638 & $23.67 \%$ \\
\hline Network news workers & 429 & $15.92 \%$ \\
\hline Network ethics and regulations & 561 & $20.82 \%$ \\
\hline Network communication education & 245 & $9.09 \%$ \\
\hline Macro and micro effects & 136 & $5.14 \%$ \\
\hline Website construction and operation & 327 & $12.13 \%$ \\
\hline Network audience & 116 & $4.30 \%$ \\
\hline Network content analysis & 67 & $2.49 \%$ \\
\hline Others & 176 & $6.53 \%$ \\
\hline Total & 2695 & $100 \%$ \\
\hline
\end{tabular}

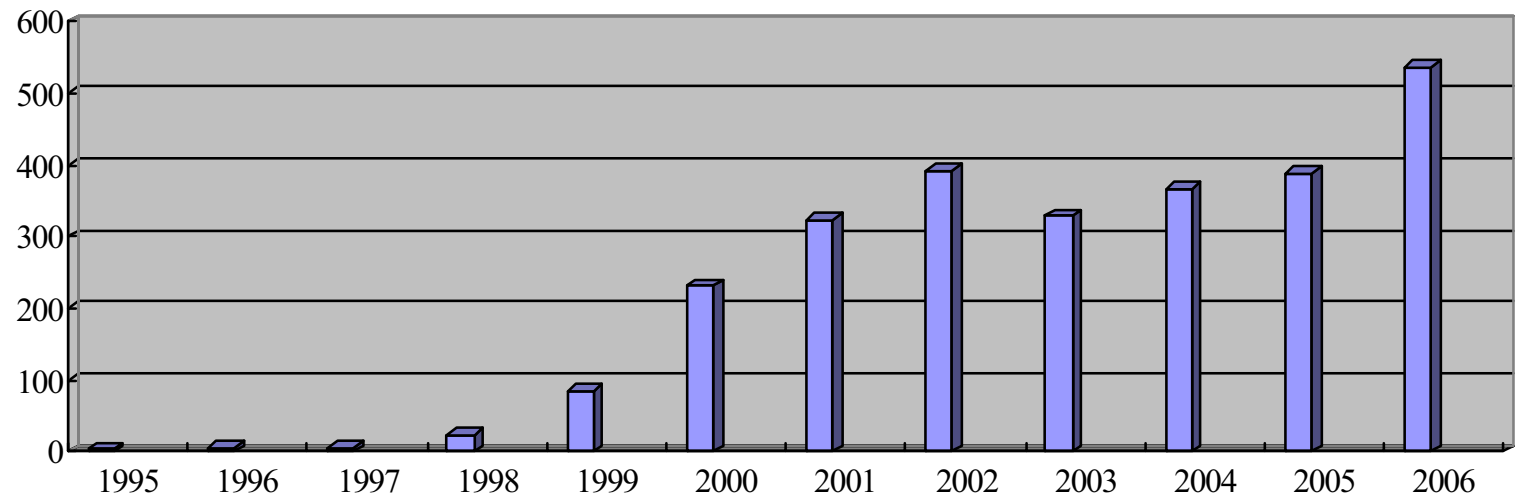

Figure 1. Papers of Network Communication Research being Published from 1995 to 2006

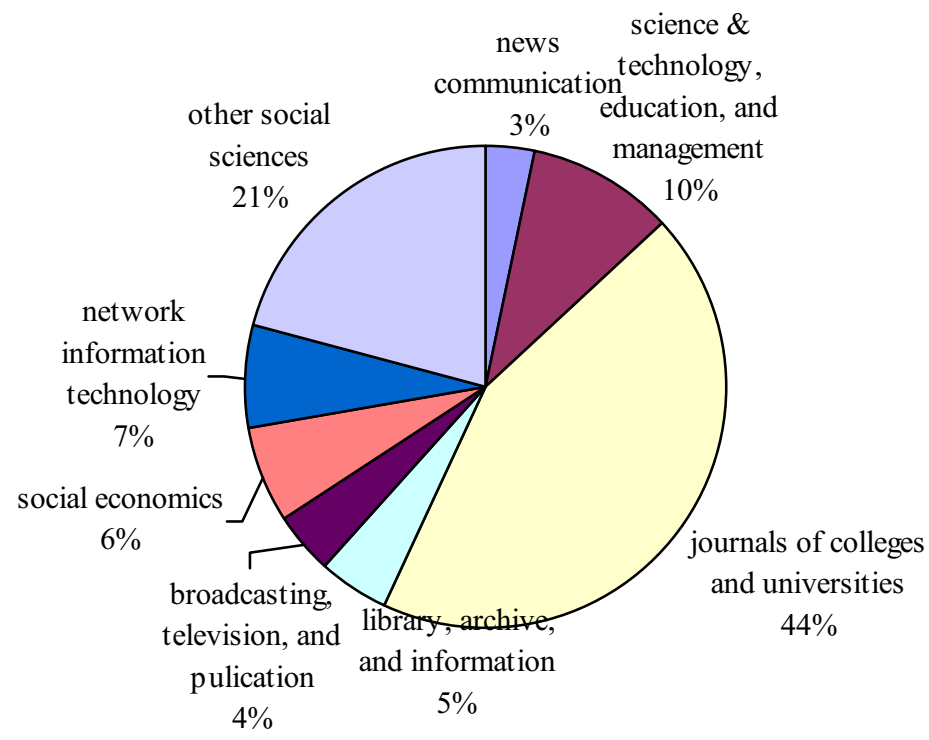

Figure 2.The Distribution of Network Communication Research Papers in Different kinds of Journals 


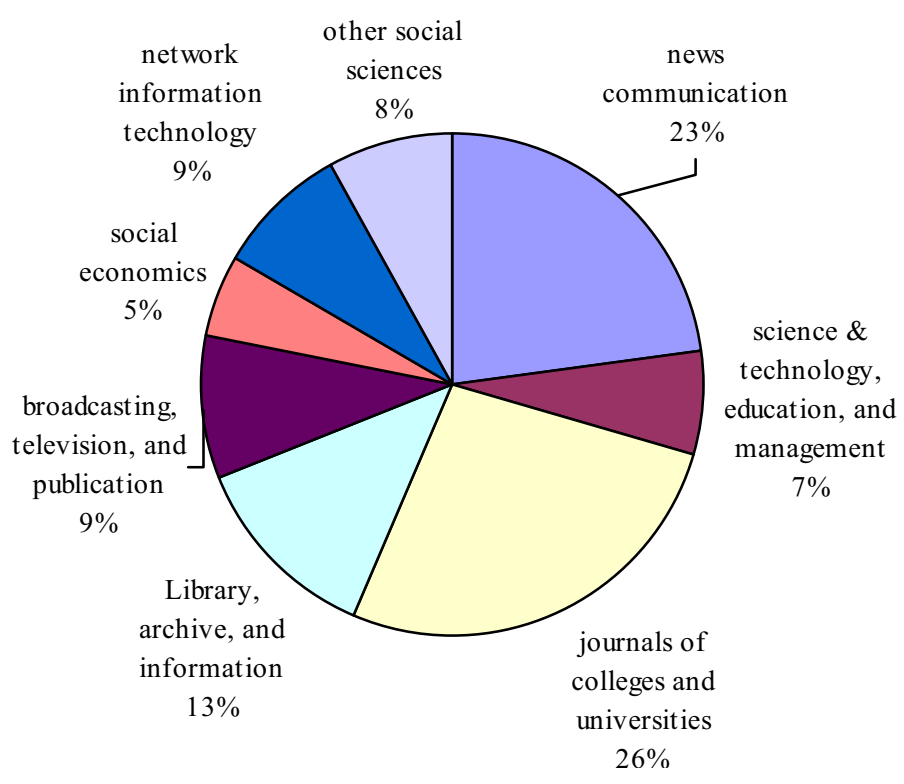

Figure 3. Numbers of Relevant Papers in Journals 